\title{
Modelos de atención en salud en enfermedades raras: revisión sistemática de la literatura*
}

\author{
Healthcare Models for Rare Diseases: A Systematic Review of the Literature \\ Modelos de atenção em saúde em doenças raras: revisão sistemática da literatura
}

Camila Quirland-Lazo

DOI: https://doi.org/10.11144/Javeriana.rgps17-34.mase

Universidad de Chile, Chile

ORCID: http://orcid.org/0000-0002-0799-3303

Camilo Castañeda-Cardona

Fecha de recepción: 28 Marzo 2017

NeuroEconomix, Colombia

Fecha de aceptación: 20 Octubre 2017

ORCID: http://orcid.org/0000-0001-6837-8809

María Alejandra Chirveches Calvache

Pontificia Universidad Javeriana, Colombia

ORCID: http://orcid.org/0000-0002-5951-719X

Alberto Aroca

Pontificia Universidad Javeriana, Colombia

ORCID: http://orcid.org/0000-0003-3727-0349

Margarita Otálora-Esteban

Hospital Universitario San Ignacio, Colombia

Pontificia Universidad Javeriana, Colombia

ORCID: http://orcid.org/0000-0003-3174-1592

Diego Rosselli ${ }^{\text {a }}$

Pontificia Universidad Javeriana, Colombia

ORCID: http://orcid.org/0000-0003-0960-9480

\section{Resumen:}

Las enfermedades de baja prevalencia requieren modelos de gestión diferentes a los de otras condiciones. Este trabajo buscó recoger las experiencias internacionales. Se realizaron búsquedas en numerosas bases de datos de literatura indexada y de documentos grises. Un panel de expertos de diferentes disciplinas revisó los resúmenes de la literatura y su posible adaptación al contexto colombiano. La búsqueda inicial arrojó 5604 referencias; la búsqueda manual adicionó 31 referencias, finalmente 78 artículos aportaron información útil para el análisis. Los resultados permiten afirmar que existen varios componentes de un modelo de gestión, estos son: políticas, legislación y aspectos administrativos; definición y codificación de enfermedades; investigación y educación; centros especializados, centros de excelencia y redes de atención; diagnóstico, tamizaje, prevención y promoción; inclusión de medicamentos huérfanos; rehabilitación y manejo paliativo; organizaciones de pacientes, grupos o redes de apoyo; y apoyo sociosanitario (inclusión laboral y educativa).

Palabras clave: enfermedades raras, medicamentos del componente especializado de los servicios farmacéuticos, organización y administración, manejo de atención al paciente.

\section{Abstract:}

Low prevalence diseases require management models different from those used in other conditions. This work was intended to gather international experiences on this issue. Searches were made in many indexed literature databases as well as in those with gray literature. A panel of experts from different disciplines checked the abstracts and their potential adaptation into the Colombian context. The initial search retrieved 5604 references and the manual search added other 31 references. At the

\footnotetext{
a Autor de correspondencia. Correo electrónico: diego.rosselli@gmail.com
} 
end, 78 articles provided useful information for the analysis. The results allow to state that a management model consists of several components, to wit: policies, legislation and administrative aspects; definition and coding of the diseases; research and education; specialized centers; excellence centers and service networks; diagnosis, screening, prevention, and promotion; orphan drug inclusion; rehabilitation and palliative care; organizations of patients and support groups or networks; and social-sanitary support (labor and educational inclusion).

Keywords: rare diseases, drugs in the pharmaceutical service specialized component, organization and administration, patient service management.

\section{Resumo:}

As doenças de baixa prevalência requerem modelos de gestão diferentes aos de outras condições. Este trabalho visou coletar experiências internacionais. Realizaram-se pesquisas em numerosos bancos de dados de literatura indexada e documentos cinza. Um painel de expertos de diferentes disciplinas revisou os resumos da literatura e sua possível adaptação no contexto colombiano. A procura inicial resultou em 5604 referências; a procura manual adicionou 31 referências, por fim 78 artigos forneceram informações úteis para a análise. Os resultados permitem afirmar que existem vários componentes de um modelo de gestão, quais são: políticas, legislações e aspetos administrativos; definição e codificação de doenças; pesquisa e ensino; centros especializados, centros de excelência e redes de atendimento; diagnóstico, triagem, prevenção e promoção; inclusão de medicamentos órfãos; reabilitação e cuidados paliativos; organizações de pacientes, grupos ou redes de apoio; e apoio sócio-sanitário (inclusão laboral e educativa).

Palavras-chave: doenças raras, medicamentos do componente especializado dos serviços farmacêuticos, organização e administração, manejo de atenção ao paciente.

\section{Introducción}

Las enfermedades raras han entrado a la agenda pública y a las discusiones de sistemas de salud en todo el mundo. En la Unión Europea se estima que existen entre 27 y 36 millones de afectados de alguna de las 6000 a 8000 enfermedades raras [1,2]; y habría otros 25 millones en Estados Unidos [3]. En Colombia, según la Federación Colombiana de Enfermedades Raras (Fecoer), podría haber alrededor de 3.8 millones de personas afectadas [4], lo que equivale a $6 \%$ a $8 \%$ de la población [2]. Sin embargo, en una consulta al Sistema Integrado de Información de la Protección Social (Sispro) se encontró que entre 2009 y 2014 hubo un total de 13173 personas registradas con algún diagnóstico de enfermedad rara, de las cuales 1199 se encuentran registradas con alguno grado de discapacidad; de ellos 503 son mujeres (42\%) y 696 hombres (58\%); el grupo más grande de pacientes se encuentra ubicado entre nueve y diecinueve años [5].

En general, se estima que $80 \%$ de las enfermedades raras tienen origen genético, $50 \%$ afectan a la población infantil y $30 \%$ de los pacientes con diagnóstico de enfermedad rara mueren antes de cumplir cinco años. Este grupo de enfermedades tiene una gran variedad de síntomas, que difieren de enfermedad a enfermedad e incluso dentro de la misma enfermedad. El impacto en la expectativa de vida y el compromiso de las habilidades físicas y mentales es muy variable $[2,6]$. La definición misma de enfermedad rara varía entre países. La OMS define como enfermedad rara a aquella con una prevalencia inferior a $0.65 \%$ o 1 \%. La Unión Europea estableció una prevalencia de menos de cinco casos por 10000 habitantes. En Estados Unidos son aquellas con una prevalencia menor de 200000 habitantes [7], mientras que Australia define enfermedades huérfanas cuando el número de afectados es menor a 2000 diagnosticados. En otros países como Taiwán la prevalencia tiene que ser menor de uno en 10000 habitantes y en Japón afecta a menos de 50000 individuos [8]. En Colombia según la legislación (Ley 1438 de 2011, que modificó el artículo 2 de la Ley 1392 de 2010), se define con una prevalencia menor de uno por cada 5000 [9].

En todos los países existen múltiples retos que enfrentan los sistemas de salud para poder implementar un sistema de atención para las enfermedades raras. Algunos son inherentes a las mismas características de estas enfermedades y tienden a ser comunes, mientras que otros dependen de las condiciones propias de cada país y de sus sistemas de salud. Entre estas dificultades se encuentran la implementación adecuada de sistemas de recolección de información sobre enfermedades raras, la investigación de calidad para el desarrollo de nuevas tecnologías o para mejorar el acceso a los servicios, las dificultades en el diagnóstico oportuno y preciso, la 
educación de todos los profesionales de la salud implicados en prevención, tratamiento y rehabilitación, la distribución de los recursos disponibles, las barreras en la inclusión de nuevos medicamentos y tratamientos muchas veces de alto costo y, finalmente, la participación de pacientes en el desarrollo de políticas públicas, investigación y desarrollo de sus propios modelos de atención $[7,10,11]$. Es claro que la atención de estas enfermedades obliga a diseñar estrategias que difieren en muchos aspectos de las que se necesitan para atender las enfermedades de alta prevalencia. Este trabajo se concentra en esas diferencias.

El objetivo fue describir los componentes y los procesos empleados en la experiencia internacional para atender las necesidades en salud de los pacientes con enfermedades raras, con el rigor que exigen los estándares internacionales.

La búsqueda estuvo orientada a construir en una etapa posterior, una propuesta de un modelo de atención para Colombia.

\section{Material y métodos}

\section{Constitución del panel de expertos}

Se conformó un panel de expertos para tratar de garantizar la calidad, la aplicabilidad y la credibilidad de la información y de las recomendaciones generadas [12]. Este panel estuvo conformado por expertos metodológicos, representantes gubernamentales y de los entes reguladores, de organizaciones de pacientes y de la comunidad científica. Su tarea incluía resolver los problemas metodológicos y de implementación que se presentaran dentro del desarrollo del proyecto [13]. Se convocó en total a cincuenta expertos de diversas áreas de las enfermedades raras. En total, treinta expertos confirmaron su participación. El panel de expertos recibió vía correo electrónico toda la metodología y el desarrollo de esta revisión sistemática, y, por medio de un método Delphi modificado, se incorporaron sus aportes en diversos puntos de la revisión. En primera instancia, se envió la información sobre la metodología implementada, y posteriormente se envió un documento de síntesis y análisis de los pilares claves para la atención de pacientes. Finalmente, se les solicitaron comentarios y recomendaciones puntuales sobre la información suministrada.

Para garantizar la transparencia, todos los miembros con la capacidad de tomar decisiones en el proceso de elaboración del proyecto y con influencia sobre su contenido declararon por escrito sus intereses [14] en un formato establecido (anexo 1) [15]. La primera tarea del panel fue validar la pregunta de investigación para establecer los criterios de búsqueda. Esta se hizo en formato PICOT (del inglés Patient, Intervention, Comparator, Outcome, Time), con énfasis en los desenlaces (outcomes) en torno a los cuales se desarrolló el proyecto [16], como muestra en la tabla 1. 
TABLA 1

Establecimiento de la pregunta de investigación por medio de la estrategia PICOT

\begin{tabular}{|c|c|c|c|}
\hline Población & Intervención & Comparador & Desenlace \\
\hline $\begin{array}{l}\text { Pacientes } \\
\text { con } \\
\text { diagnóstico } \\
\text { de } \\
\text { enfermedad } \\
\text { rara en } \\
\text { cualquier } \\
\text { pais }\end{array}$ & $\begin{array}{l}\text { Modelos, planes, estrategias o } \\
\text { elementos empleados en la } \\
\text { atención de enfermedades raras: } \\
\text { politicas y legislación, definición } \\
\text { y codificación, investigación y } \\
\text { educación, centros especializados, } \\
\text { centros de excelencia y redes de } \\
\text { atención especifica, diagnóstico y } \\
\text { tamizaje, inclusión de } \\
\text { medicamentos huérfanos, } \\
\text { rehabilitación, organizaciones de } \\
\text { pacientes, apoyo sociosanitario, } \\
\text { aspectos administrativos, factores } \\
\text { facilitadores, barreras, limitantes }\end{array}$ & $\begin{array}{l}\text { Comparación } \\
\text { con statu quo } \\
\text { o entre } \\
\text { modelos, } \\
\text { planes, } \\
\text { estrategias y } \\
\text { protocolos }\end{array}$ & $\begin{array}{l}\text { Impacto en } \\
\text { resultados: politicas } \\
\text { y legislación, } \\
\text { investigación, } \\
\text { educación, centros de } \\
\text { excelencia, redes de } \\
\text { atención, acceso en } \\
\text { atención } \\
\text { medicamentos, } \\
\text { sostenibilidad del } \\
\text { sistema, aspectos } \\
\text { sociales }\end{array}$ \\
\hline
\end{tabular}

\section{Estrategia de búsqueda}

Se realizó una búsqueda sistemática de la literatura para la pregunta de investigación planteada, que cumpliera criterios iniciales de baja especificidad y alta sensibilidad, enfocada en términos libres y palabras clave [13]. Las bases de datos y términos utilizados para la búsqueda se muestran en la tabla 2. 
TABLA 2

Bases de datos y términos utilizados para la búsqueda

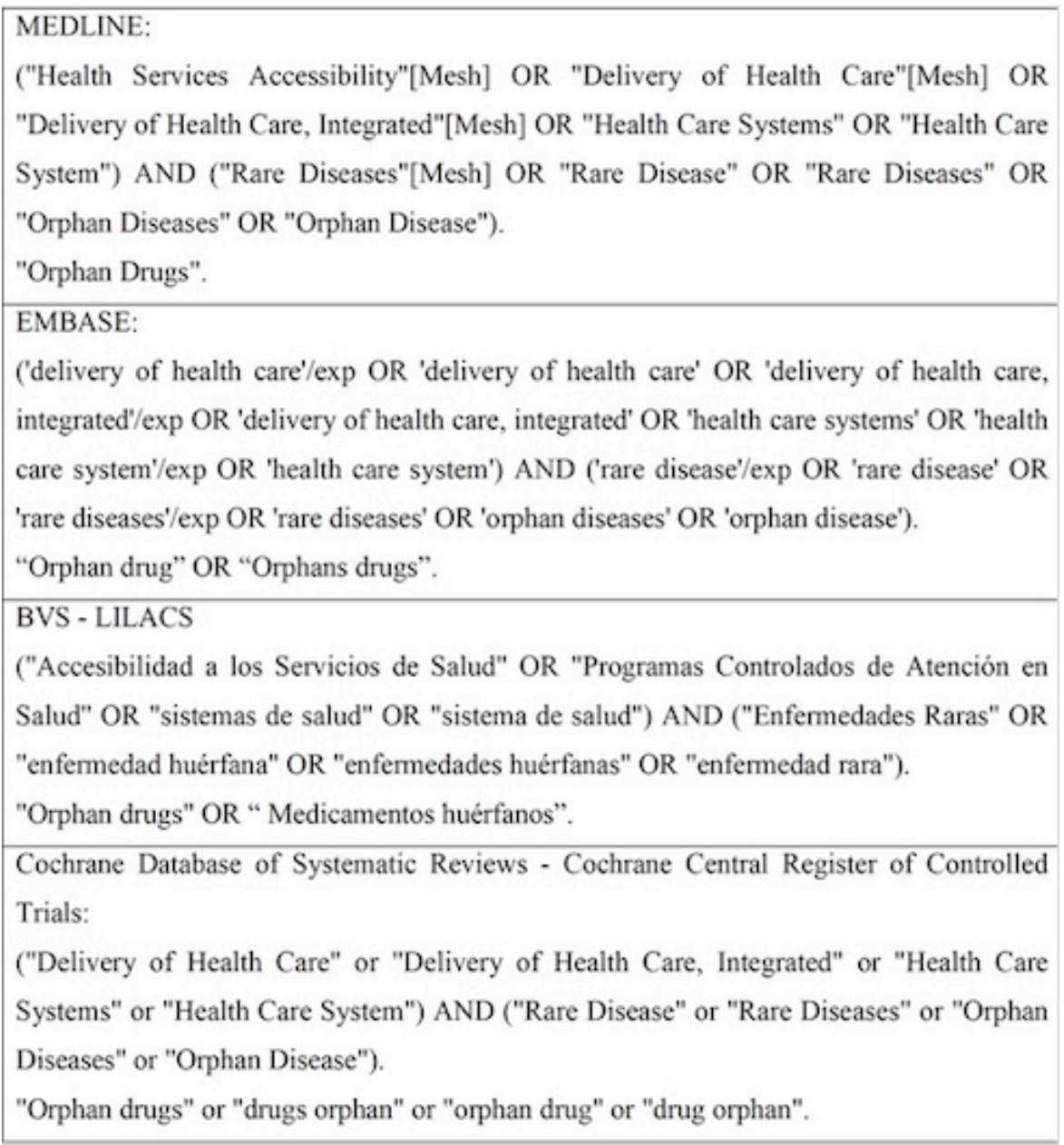

De manera paralela, se realizaron también búsquedas en otras bases de datos específicas de enfermedades raras como Orphanet, Genetic and Rare Diseases Information Center (GARD), Disease InfoSearch, páginas gubernamentales como Eurordis, Organización Nacional de Enfermedades Raras (NORD) y Federación Colombiana de Enfermedades Raras (Fecoer). También se buscó literatura publicada en revistas no indexadas empleando buscadores como Google Académico, en memorias de congresos, documentos publicados por sociedades científicas y en las listas de referencias de todos los artículos. Adicionalmente, se consultaron páginas gubernamentales que presentaran modelos, planes, políticas y estrategias ya establecidas para diferentes países del mundo [17]. 


\section{Selección de referencias, extracción y análisis de la información}

Con los artículos obtenidos a partir de las búsquedas, se aplicaron criterios de inclusión y exclusión preestablecidos [18]. Se revisó la literatura en español, inglés, italiano, alemán, francés y portugués, incluyendo revisiones sistemáticas y metaanálisis, estudios primarios, estudios descriptivos, revisiones narrativas, planes, estrategias o proyectos gubernamentales. Se incluyeron artículos que se refirieran a cualquiera de los diferentes aspectos organizativos, desde programas de tamizaje a cuidados paliativos, o de aspectos legales, políticos y económicos referentes a este grupo de enfermedades. Solo se excluyeron artículos de opinión que no trajeran información factual.

Seguidamente se aplicó un primer filtro, según el título y el resumen (abstract), para excluir los que fueran claramente irrelevantes. Los documentos grises incluidos fueron aquellos que plantearan una metodología de trabajo y que tuvieran un sustento bibliográfico. El segundo filtro consistió en evaluar la calidad de los textos completos por medio de la herramienta Notari, un instrumento para la evaluación de cualitativa de textos narrativos y de opinión, desarrollado por el Joanna Briggs Institute, de la Universidad de Adelaida, en Australia [19]. Este proceso de evaluación se realizó de manera pareada. Los desacuerdos se resolvieron por consenso, invitando a un tercer integrante del equipo de trabajo.

Posteriormente, con una lista de los artículos seleccionados para la extracción se realizó un resumen individual, según el enfoque o componente que cada estudio aportara al modelo integral y una agrupación por categorías de información. Se elaboraron también documentos de síntesis que fueron presentados al panel de expertos, para que en consenso se hiciera un análisis de los resultados, se generaran preguntas adicionales sobre las lecturas y se estudiaran las conclusiones y recomendaciones.

\section{Resultados}

La búsqueda sistemática sobre modelos, planes y estrategias en enfermedades raras arrojó un total inicial de 5604 referencias, 179 de ellas fueron duplicadas y 5330 artículos fueron excluidos por irrelevantes. La búsqueda manual adicionó 31 referencias, se totalizaron 126 publicaciones para revisar en texto completo; no se consiguieron 13 referencias y en un segundo filtro se excluyeron 35 publicaciones. Finalmente, 78 artículos aportaron información útil para el análisis (figura 1). Estos documentos cuentan la experiencia de un total de 41 estados, provincias, países, o regiones del mundo. 


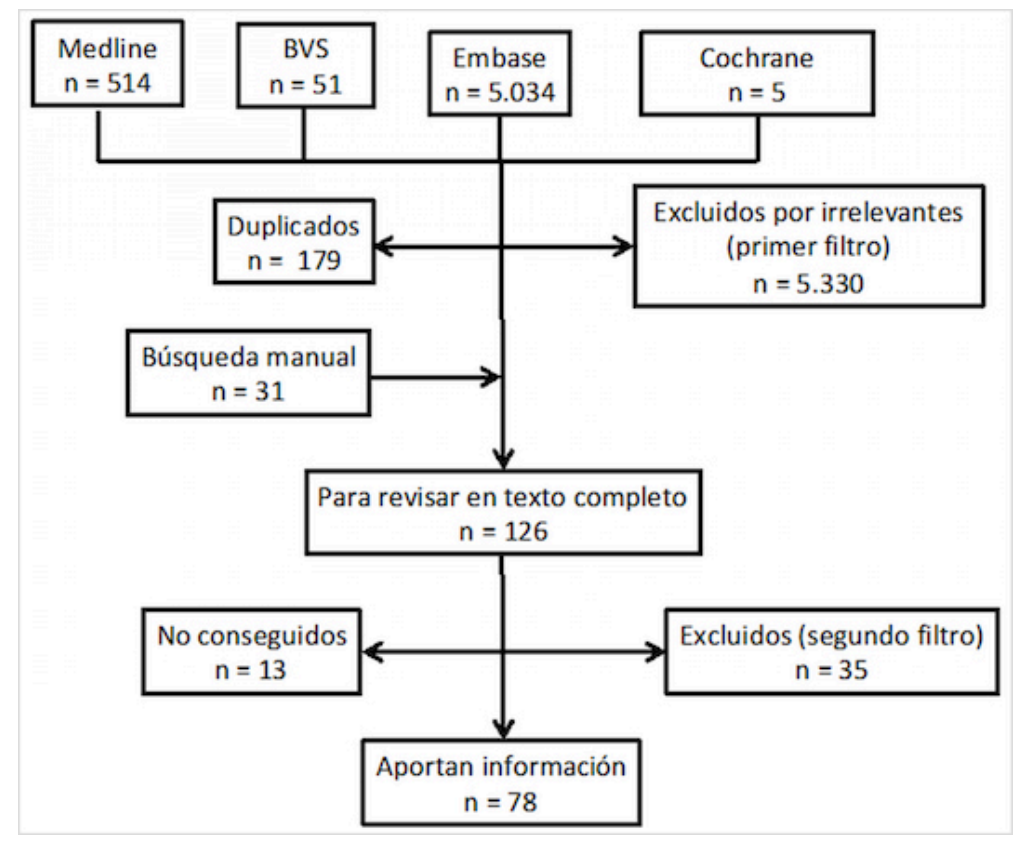

FIGURA 1

Prisma - modelos, planes y estrategias en enfermedades raras

Al tratar de agrupar los artículos y documentos en categorías, se llegó a los siguientes nueve componentes o pilares: políticas, legislación y aspectos administrativos; definición y codificación; investigación y educación; centros especializados, centros de excelencia y redes de atención; diagnóstico, tamizaje, prevención y promoción; inclusión de medicamentos huérfanos; rehabilitación y manejo paliativo; organizaciones de pacientes, grupos o redes de apoyo; y, finalmente, apoyo sociosanitario, con aspectos como inclusión laboral y educativa.

De cada uno de estos pilares se hicieron resúmenes y recomendaciones, con aportes del panel de expertos y se redactó un documento final que ha sido puesto en consideración del Ministerio de Salud y Protección Social, de los aseguradores y prestadores, de la academia, de las organizaciones de pacientes y del público en general.

\section{Discusión}

El trabajo aquí presentado corresponde a una metodología que combina una revisión sistemática de la literatura basada en las directrices internacionales que muestra información sobre enfermedades raras de 41 organizaciones de estados, provincias, países, o regiones del mundo.

La conformación del panel de expertos permitió garantizar la calidad, la aplicabilidad y la credibilidad de la información y de las recomendaciones generadas [12], que ya de por sí el diseño del estudio da a los resultados arrojados.

No solo se tuvo en cuenta la literatura hallada en la revisión sistemática, sino que se incluyó literatura gris obtenida por búsqueda manual de bases de datos específicas y literatura no indexada. Debido a la gran cantidad y variedad de información obtenida, se decidió agruparla y organizarla en nueve componentes, lo cual facilita su comprensión. Esta agrupación de la formación por componentes no fue caprichosa, sino que 
fue replicada al identificarse en los diferentes modelos y planes nacionales para enfermedades raras hallados en la búsqueda. No hubo limitaciones en cuanto a los idiomas de las referencias encontradas, ya que revisó literatura en español, inglés, italiano, alemán, francés y portugués. Sin embargo, el estudio presenta ciertas limitaciones. Una de estas es el sesgo de publicación por la gran cantidad de referencias encontradas que pudo llevar a desestimar ciertos resultados. La inclusión de literatura gris y no indexada puede restar calidad y credibilidad a los resultados obtenidos, sin embargo, la rigurosidad del panel de expertos y de la evaluación de la calidad la información por medio de la metodología del estudio las garantiza.

Este estudio es muy importante, por ser el primero de este tipo realizado en Colombia, y también en Latinoamérica, así como por permitir entender las necesidades en salud de los pacientes con enfermedades raras en otras partes del mundo y poder así proponer un modelo de atención para enfermedades raras en Colombia.

\section{Conclusión}

La información obtenida a través de los resultados del presente estudio permitió elaborar los nueve componentes que constituyen los pilares fundamentales en la elaboración e implementación de los modelos, planes, políticas o estrategias para pacientes con diagnóstico de enfermedades raras. A partir de esta metodología común, cada uno de los componentes estudiados será publicado individualmente, con el fin de proponer un modelo global e integrado de atención para pacientes con enfermedades raras en Colombia.

\section{Referencias}

1. Organización Mundial de la Salud. Coming together to combat rare diseases. Bull World Health Organ. 2012; 90(6):406-7.

2. Montserrat A, Waligóra J. The European Union policy in the field of rare diseases. Public Health Genomics. 2013;16(6): 268-77.

3. Schieppati A, Henter J, Daina E, Aperia A. Why rare diseases are an important medical and social issue. Lancet. 2008; 371(9629):2039-41.

4. Federación Colombiana de Enfermedades Raras. Una nueva esperanza para las víctimas de enfermedades raras en Colombia [internet] [acceso: 7 de julio de 2015]. Disponible en: http://www.fecoer.org/una-nueva-esperanzapara-las-victimas-de-enfermedades-raras-en-colombia/

5. Sistema Integrado de Información de la Protección Social (Sispro) [internet] [acceso: 16 de octubre de 2015]. Disponible en: http://www.sispro.gov.co

6. Taruscio D, Trama A, Stefanov R. Tackling rare diseases at European level: why do we need a harmonized framework? Folia Med. 2007; 49(1-2):59-67.

7. Forman J, Taruscio D, Llera V. The need for worldwide policy and action plans for rare diseases. Acta Paediatr. 2012; 101(8):805-7.

8. International Conference on Rare Diseases \& Orphan Drugs. The Yukiwariso Declaration. 7th International Conference on Rare Diseases \& Orphan Drugs, Tokyo, 2012.

9. Reforma del Sistema General de Seguridad Social en Salud. Ley 1438 de enero 19 de 2011. Diario oficial 47957 de enero 19 de 2011.

10. Toumi M, Pashos C, Korchagina D, Redekop K, Morel T, Blanchette C, et al. Challenges in assessing and appraising rare disease diagnostics \& treatments. International Society for Pharmacoeconomics and Outcomes Research Rare Disease Special Interest Group; 2015.

11. Martínez Carmona MR. Las enfermedades raras y los vacíos jurídicos en la aplicabilidad de la legislación colombiana para su tratamiento [tesis de grado]. Manizales: Facultad de Ciencias Jurídicas, Universidad de Manizales; 2013. 
12. Fretheim A, Schünemann HJ, Oxman AD. Improving the use of research evidence in guideline development: 3 . Group composition and consultation process. Health Res Policy Syst. 2006; (4).

13. Instituto de Evaluación Tecnológica en Salud. Manual metodológico para la elaboración de evaluaciones de efectividad, seguridad y validez diagnóstica de tecnologías en salud. Bogotá: Instituto de Evaluación Tecnológica en Salud - IETS; 2014.

14. Boyd EA, Bero LA. Improving the use of research evidence in guideline development: 4. Managing conflicts of interests. Health Res Policy Syst. 2006; (4).

15. Centro de Estudios e Investigación en Salud (CEIS). Guía metodológica para la elaboración de guías de práctica clínica con evaluación económica en el sistema general de seguridad social en salud colombiano: versión completa final. Bogotá: Fundación Santa Fe de Bogotá - Centro de Estudios e Investigación en Salud; 2014.

16. Fretheim A, Schünemann HJ, Oxman AD. Improving the use of research evidence in guideline development: 5. Group processes. Health Res Policy Syst. 2006; (4).

17. Henderson LK, Craig JC, Willis NS, Tovey D, Webster AC. How to write a Cochrane systematic review. Nephrology. 2010; 15(6):617-24.

18. National Institute for Health and Care Excellence. The guidelines manual. Londres: National Institute for Health and Care Excellence; 2012.

19. Guyatt GH, Oxman AD, Sultan S, Glasziou P, Akl EA., Alonso-Coello P, et al. GRADE guidelines: 9. Rating up the quality of evidence. J Clin Epidemiol. 2011; 64(12):1311-6.

\section{Notas}

* Artículo de investigación.

Licencia Creative Commons CC BY 4.0

Como citar este artículo: Quirland-Lazo C, Castañeda-Cardona C, Chirveches Calvache MA, Aroca Al, Otálora-Esteban M, Rosselli D. Modelos de atención en salud en enfermedades raras: revisión sistemática de la literatura. Rev Gerenc Polít Salud. 2017; 17(34): 1-9. https://doi.org/10.11144/Javeriana.rgps17-3 4.mase 